\title{
The ISBER 2018 Awards
}

\author{
Tamsin Tarling,, Zisis Kozlakidis, ${ }^{2, *}$ Brent Schacter, ${ }^{3}$ and Sheila O’Donoghue ${ }^{4}$
}

$\mathrm{T}$ He International Society for Biological and Environmental Repositories (ISBER) will confer a number of different awards at its annual meeting this year in Dallas, Texas, USA. These include the Travel Awards, which have increased from one to four per year, as well as a number of awards celebrating the achievements and contributions of individuals from a wide range of professional backgrounds and geographical areas who have made a significant contribution to ISBER and the biobanking field in general.

\section{The ISBER Travel Awards}

The ISBER Travel Award covers all costs for individuals from low and middle income countries who are planning or currently managing a repository to attend the ISBER Annual Meeting. Historically, the ISBER Board of Directors has funded one award per year but this year a decision was made to offer the opportunity to four individuals to reflect the society's ongoing commitment to sharing biobanking knowledge globally.

The four successful individuals receiving this year's travel award:

(1) Milcah Dhoro from the University of Zimbabwe, Harare, Zimbabwe

(2) Birendra Yadav from the National Liver Disease Biobank, New Delhi, India

(3) Jean Marie Nana from Laboratoires d'Analyses Biomédicales La Grace Biobanking, Yaoundé, Cameroon

(4) Amany Mohamed Maher from the Ain Shams University, Cairo, Egypt.

All four recipients come from countries where there is a growing need for biobanking and the opportunity to attend the ISBER conference will allow them to learn more about the implementation of biobanks, collaborate with biobanking experts from around the world, educate their colleagues, and build the biobanking capacity within their institutions.

\section{The ISBER Annual Awards}

The ISBER Award for Outstanding Achievement in Biobanking, sponsored by Worthington Industries, is designed to recognize individuals who have made outstanding contributions to the field of biobanking. The award can be given for a single outstanding achievement or for a life-time body of outstanding work in the field. ISBER is proud to announce the 2018 winner of the ISBER Outstanding Achievement in Biobanking award, Dr. Jane Carpenter. Dr. Carpenter has many years' experience working in diagnostic laboratories in the United Kingdom and Australia specializing in hematology. She made the move into tissue banking in 2003, being employed by the pediatric tumor bank at Westmead Children's Hospital. Since 2006 she has been the manager of the Australian Breast Cancer Tissue Bank (ABCTB), headquartered in the Westmead Millennium Institute in NSW and has significant experience in many aspects of tissue banking both at a practical and managerial level. She has recently been appointed to the University of Sydney, Faculty of Health Sciences. Dr. Carpenter has been involved extensively in realizing educational opportunities and a career structure for tissue banking personnel staff and has been involved in the creation of an educational programme leading to a formal qualification in Tissue Banking for technical staff. Additionally, she led on some of the most innovative and extensive public outreach biobanking campaigns in Australia, raising the public understanding of the field and need of biobanking.

\footnotetext{
${ }^{1}$ Department of Pathology and Laboratory Medicine, University of British Columbia, Vancouver, Canada.

${ }^{2}$ Division of Infection and Immunity, University College London, London, United Kingdom.

${ }^{3}$ Department of Medicine/Medical Oncology and Hematology, University of Manitoba/CancerCare Winnipeg, Canada.

${ }^{4}$ Biospecimen and Biobanking Research Services, BC Cancer/UBC, Vancouver, Canada.

*ISBER President.
} 
Dr. Carpenter has been a member of the Australasian Biospecimen Network Association (ABNA) Committee since incorporation of the association in 2009 and served as Vice President and President of ABNA. She was elected to the ISBER Board of Directors (2011-17) and has been a member of the ISBER Education and Training committee from (2008-17). Jane was the co-chair of the ISBER Program Committee for the 2013 Annual Meeting held in Sydney, Australia. She is currently a member of the Asian Network of Research Resource Centres (ANRRC) Board of Directors and a member of the Marble Arch International Working Group on Biobanking.

The ISBER Founder's Award, sponsored by Chart MVE, recognizes individuals who have provided outstanding leadership to the founding, support, and incorporation of ISBER as an international biobanking society. ISBER is proud to announce Dr. Jim Vaught as the 2018 recipient of the ISBER founder's award. Dr. Vaught spent 14 years at the U.S. National Cancer Institute (NCI), most recently as the Chief of the Biorepositories and Biospecimen Research Branch (BBRB) in the Cancer Diagnosis Program. He has been working in the field of biorepository and biospecimen science for over 20 years. In 1999, he was one of the founding members of the ISBER and was its second President, and elected President for a second time in 20152016. In addition to ISBER, Dr. Vaught is a member of the Board of Directors of the National Disease Research Interchange, of the American Association for Cancer Research, the American Society for Pharmacology and Experimental Therapeutics, and the American Association for Clinical Chemistry. In 2012 Dr. Vaught became Editorin-Chief of Biopreservation and Biobanking, the official journal of ISBER. He is currently a Senior Research Fellow at the International Prevention Research Institute (Lyon) and a consultant for a number of international biobanking activities.

The ISBER Distinguished Leadership and Service Award is designed to honor ISBER members who have demonstrated exceptional leadership to further the mission and goals of the society and/or significant, long-standing contributions to the society. ISBER is proud to announce Dr. Lori Campbell as the 2018 recipient of the ISBER Distinguished Leadership and Service Award. Dr. Campbell has been a member of ISBER since 2003, member of the Education and Training Committee (2004-10), of the Publications Committee (2011-15) and of the Board of Directors (2011-14). Dr. Campbell has been the Associate Editor of the ISBER Best Practices for Repositories first and second edition (2004 and 2008 respectively), and Editor-in-Chief of the ISBER Best Practices third and fourth edition (2012 and 2018). ISBER's Best Practices for Repositories has been recognized globally as the premier handbook for biobanking. It provides a comprehensive tool to guide repository professionals in the technical, legal, ethical, and/or managerial issues relevant to repositories of biological and environmental specimens. Dr Campbell's contribution was not limited to the development and editing of the document, but extended to the central coordinator for all communication to and from stakeholders, submitting appropriate drafts to ISBER committee and Board of Directors for approval, and drafting guidelines for the continuous development of the ISBER Best Practices for Repositories.
The ISBER Special Service Awards recognize individuals who have made exceptional contributions toward the goals of the society through the performance of a special service or act on behalf of the organization. ISBER is proud to announce the following recipients of the 2018 ISBER Special Services Award:

- Prof. Xiaomin Wang receives this award for his exemplary leadership in developing the field of biobanking in China, his contribution to the biospecimen research science, and for his services to ISBER as Director at Large for China (2013-17).

- Dr. Andy Brooks receives this for being a most active representative for ISBER globally both academically and business-wise throughout 2017-18. His successful combination of an academic and business career is combined with a unique long-term commitment and promotion of the applied side of the science of biobanking, uniquely focusing on developing biobanking as a science and a business.

- Prof. Koh Furuta receives this award for his long-term contribution to biobanking in Japan, the creation of the Council of Industrial use of Biological and Environmental Repositories (CIBER) in Japan in 201718 and his leadership in the ISBER ISO/TC276/WG2 Task-force, contributing in this way to the new era of standardization.

- Mark Barnes is also a distinguished recipient of the special services award. Mr. Barnes is an ISBER member and partner with the law firm Ropes and Gray. He serves on the US DHHS Secretary's Advisory Committee for Human Research Protections and has played a major role in ensuring that policy and regulations in the United States regarding human specimen research and biobanking are rational and that they protect research participants while at the same time facilitating important specimen research. He has shared his time, unique legal expertise, and experience by presenting at numerous ISBER Annual Meetings and workshops. In addition, he played a critical role in 2017 in developing and refining ISBER's public education Q\&As in preparation for the media inquiries in response to the release of the Henrietta Lacks movie.

- Dr. Marianne Henderson is a recipient of the special services award for her unwavering resolve to leading the creation of a series of successful ISBER meetings in 2017-18 as Chair of the ISBER Organising Advisory Committee and for her educational biobanking activities in Low- and Middle-Income Countries globally.

- Dr Helen Morrin receives this award for her critical role in co-chairing the ISBER Science Policy Advisory Committee and public education initiatives for many years. Furthermore, she led the effort to revise the ISBER Science Policy webpages in 2017, leading to major improvements and serving as a model for future efforts to improve the ISBER website.

- Ms. Marianna Bledsoe is a recipient of the special services award. Mr. Bledsoe chairs the Science Policy Committee that is one of the most active ISBER committees. The committee coordinates responses to 
the important ethical and regulatory issues facing our membership. This past year, Marianna and her colleagues on the committee coordinated a comprehensive response to a request for comments on the Proposed Changes to Canadian Tri-Council Policy Statement (TCPS 2, 2014) Guidance for Research involving $\mathrm{Hu}-$ man Cells and Cell Lines. She has also played a critical role in the ISBER ISO/TC276/WG2 Task force and the preparation for the media inquiries in response to the release of the Henrietta Lacks movie.

- Mr. Xuexun Zhou from Avantech, Shanghai, China. Mr. Zhou has been a tireless supporter of ISBER, showcasing the evidence base in the biobanking field in China and acting as a central liaison between ISBER members in China and globally. This award is in recognition of his services in the research on biobanking sustainability in China in 2017, as well as for his support in the preparation of the 2019 ISBER Annual Meeting and Exhibits in Shanghai, China.

These awards will be presented to the recipients at the ISBER business meeting in Dallas on May 23, 2018. We hope you will join us in congratulating these very deserving recipients of these prestigious awards. Congratulations to these outstanding awardees.

Address correspondence to: Zisis Kozlakidis, PhD, MBA Division of Infection and Immunity University College London WC1E 6BT, London

United Kingdom

E-mail: z.kozlakidis@ucl.ac.uk 